\title{
Role of P2X7R in eosinophilic and non-eosinophilic chronic rhinosinusitis with nasal polyps
}

\author{
YAN WANG $^{1 *}$, SHAN CHEN $^{1 *}$, WENWEN WANG ${ }^{1}$, JIANJUN CHEN $^{1}$, WEIJIA KONG $^{1,2}$ and YANJUN WANG ${ }^{1,2}$ \\ ${ }^{1}$ Department of Otorhinolaryngology, Union Hospital, Tongji Medical College; ${ }^{2}$ Institute of Otorhinolaryngology, \\ Union Hospital, Tongji Medical College, Huazhong University of Science and Technology, Wuhan, Hubei 430022, P.R. China
}

Received November 30, 2020; Accepted April 14, 2021

DOI: $10.3892 / \mathrm{mmr} .2021 .12160$

\begin{abstract}
Chronic rhinosinusitis with nasal polyps (CRSwNP) is an inflammation-mediated disease of the nasal mucosa. P2X7R has been reported to be a potential biomarker for inflammation. The aim of the present study was to explore the role of P2X7R in CRSwNP, and the interaction between P2X7R and the NLRP3 inflammasome in the development of CRSwNP. Firstly, the expression profiles of P2X7R in nasal mucosa were investigated using western blotting (WB), polymerase chain reaction (PCR) and immunofluorescence (IF) staining. Next, the effect of inflammatory stimulation with lipopolysaccharides (LPS) combined with 2'(3')-O-(4-benzoylbenzoyl) adenosine 5'-triphosphate triethylammonium salt (BzATP) on primary human nasal epithelial cells (HNECs) was determined. Then, the therapeutic effect of the selective P2X7R antagonist, A740003, on P3X7R, NOD-like receptor pyrin domain containing 3 (NLRP3) inflammasome and IL-1 $\beta$ alterations in HNECs was explored using enzyme-linked immunosorbent assay, WB and PCR. It was found that P2X7R was overexpressed in CRSwNP, especially in eosinophilic CRSwNP, the expression of P2X7R, NLRP3 and IL-1 $\beta$ were upregulated in HNECs after induction by LPS combined with BzATP; but the expression of NLRP3 and IL-1 $\beta$ were downregulated after stimulation with A740003. The aforementioned results indicate that P2X7R-mediated NLRP3 inflammasome activation may have a role in the pathogenesis of CRSwNP.
\end{abstract}

Correspondence to: Dr Weijia Kong, Institute of Otorhinolaryngology, Union Hospital, Tongji Medical College, Huazhong University of Science and Technology, 1277 Jiefang Avenue, Wuhan, Hubei 430022, P.R. China

E-mail: entwjkong@hust.edu.cn

Dr Yanjun Wang, Department of Otorhinolaryngology, Union Hospital, Tongji Medical College, Huazhong University of Science and Technology, 1277 Jiefang Avenue, Wuhan, Hubei 430022, P.R. China

E-mail: yjwang@hust.edu.cn

*Contributed equally

Key words: chronic rhinosinusitis with nasal polyps, NLRP3, $\mathrm{P} 2 \mathrm{X} 7$, human nasal epithelial cells, eosinophilic

\section{Introduction}

Chronic rhinosinusitis with nasal polyps (CRSwNP) is a nasal inflammatory disease, characterized by symptoms including nasal obstruction, drainage, smell loss and facial pain or pressure (1). CRSwNP affects a large proportion of the population world-wide and is associated with high cost of management and low quality of life (2). The prevalence of CRSwNP in Europe is estimated to be between 2.1 and $4.4 \%$, while it is $4.2 \%$ in the United States and $1.1 \%$ in China (3). Lourijsen et al (4) found that the total direct costs were 1,501 Euros per year per patient with CRSwNP. CRS is currently classified into eosinophilic CRS (ECRS) and non-ECRS (NECRS) subtypes based on the presence or absence of tissue eosinophilic infiltration (1). Therefore, CRSwNP may also be subclassified into eosinophilic CRSwNP (ECRSwNP) and non-eosinophilic CRSwNP (NECRSwNP) (5), with the former having a higher recurrence rate and asthma incidence (6). Over the past 40 years, advances in functional endoscopic sinus surgery and pre- and postoperative drug therapy have greatly improved the cure rate of CRSwNP; however, disease recurrence ranges from 40 to $78.9 \%$ in CRSwNP, and the rate of revision surgery is as high as $36.8 \%(7,8)$. There is still a lack of effective mechanism-based treatments in clinical practice.

NOD-like receptor pyrin domain containing 3 (NLRP3) can assemble with apoptosis-associated speck-like protein containing a CARD (ASC) and pro-caspase-1 to form a multimeric protein complex called the NLRP3 inflammasome. Recently, the NLRP3 inflammasome was demonstrated to be implicated in the pathogenesis of CRSwNP (9). It was found that the NLRP3 inflammasome was activated in nasal mucosa in a murine acute bacterial rhinosinusitis model (10). This suggested that the NLRP3 inflammasome contributed to nasal inflammation. Activated caspase-1, generated when pro-caspase-1 is cleaved by the NLRP3 inflammasome, proteolytically cleaves the inflammatory cytokines IL-1 $\beta$ and IL-18 into their mature forms (11). Elevated IL-1 $\beta$ and IL-18 levels are found in nasal polyps of CRSwNP patients $(9,12)$. Furthermore, increased IL-18 levels was significantly associated with the radiological severity of sinusitis and local eosinophilia (12). Therefore, a mechanism for decreasing the levels of inflammatory cytokines may provide a clinical benefit. The formation of the NLRP3 inflammasome can be induced by several microbial and nonmicrobial stimuli. 
Nonmicrobial stimuli include substances which cause specific diseases such as uric acid, silica fibres and extracellular adenosine triphosphate (ATP) $(13,14)$. The purinergic $2 \times 7$ receptor $(\mathrm{P} 2 \mathrm{X} 7 \mathrm{R})$ can be triggered by ATP which then results in the assembly of the NLRP3 inflammasome (15).

The ATP-gated ionotropic $\mathrm{P} 2 \mathrm{X}$ receptor subfamily consists of seven (1-7) members, and several $\mathrm{P} 2 \mathrm{X}$ receptors, including $\mathrm{P} 2 \mathrm{X} 7$, are expressed in normal human nasal epithelial cells (16). An increased ATP concentration induced by infection or inflammation can act on the P2X7 receptor leading to NLRP3 inflammasome and caspase-1 activation (17). However, the specific role of $\mathrm{P} 2 \mathrm{X} 7 \mathrm{R}$ in CRSwNP has not yet been established, and the interaction between P2X7R and the NLRP3 inflammasome in the development of CRSwNP remains unclear. Therefore, it was hypothesized that $\mathrm{P} 2 \mathrm{X} 7 \mathrm{R}$ is upregulated in nasal polyps and modulation of the P2X7R/inflammasome axis would attenuate lipopolysaccharide (LPS)-induced inflammation in cultured human nasal epithelial cells.

\section{Materials and methods}

Patients. A total of 32 patients with CRSwNP (16 ECRSwNP and 16 NECRSwNP) and 16 control subjects were included in the present study. NP specimens were obtained from patients diagnosed with CRSwNP according to the criteria of the European Position Statement updated in 2020 (1). Human nasal mucosa of the middle turbinate were collected from patients that underwent neurosurgery, who had undergone surgery because of a pituitary tumour, as the control. The visual analogue scale and computed tomography scores were graded according to the method previously described (18). The clinical characteristics of patients are listed in Table I. Subjects who had used oral or nasal corticosteroids, anti-histamines, antibiotics or antileukotrienes within the preceding 4 weeks before sample collection were excluded. The collected samples were used for haematoxylin-eosin (HE) staining, western blotting (WB), immunofluorescence (IF) staining, and primary human nasal epithelial cell (HNECs) culture. The present experimental study was approved by the ethical committees of Tong-ji Medical College, Huazhong University of Science and Technology (permit no. S135). All participants of the study were informed and signed a consent form.

Histological and immunofluorescence observation. The NP tissues collected from patients were immediately fixed overnight in $4 \%$ formaldehyde-phosphate buffered saline solution, then dehydrated through a graded ethanol series (70, 80, 90, 95 and 100\%) for 5 min each, before samples were embedding in paraffin, and finally sectioned at 5- $\mu \mathrm{m}$ thickness. In order to quantify the eosinophilic infiltration of NP, haematoxylin and eosin staining was performed. The NP sections were observed at high power (HP) (magnification, x400; Leica $\mathrm{GmbH}$; cat. no. DM2500) and then, $10 \mathrm{HP}$ fields were randomly selected and eosinophil numbers were microscopically counted. A tissue eosinophil count of 10 or more eosinophils per high-power field (HPF) was defined as ECRSwNP $(1,19)$. After deparaffinization and rehydration, the tissue sections underwent heat-induced epitope retrieval followed by blocking with $10 \%$ bovine serum albumin protein at room temperature for $30 \mathrm{~min}$. The blocked sections were incubated with rabbit anti-human P2X7R (1:200; GeneTex; cat no. GTX104288) at $4^{\circ} \mathrm{C}$ overnight. The next day, the sections were rinsed three times with PBS, and incubated with secondary anti-rabbit antibody (1:300; antGene; cat no. ANT032) in the dark at room temperature for $1 \mathrm{~h}$ and counterstained with DAPI (Beyotime Institute of Biotechnology; cat. no. C1005). Images were captured with a confocal laser scanning microscope (Nikon-A1-Si; Nikon Corporation).

Cell culture.HNECs isolated from seven patients with CRSwNP were cultured according to a previously reported method (9). In brief, the tissues were transferred and digested with $0.1 \%$ protease from Streptomyces griseus (cat no. 9036-06-0; Sigma-Aldrich; Merck KGaA) and $0.1 \mathrm{mg} / \mathrm{ml}$ deoxyribonuclease (cat no. D5025; Sigma-Aldrich; Merck KGaA). Separated epithelial cells were collected and seeded onto PureCol $^{\mathrm{TM}}$ EZ Gel solution (Sigma-Aldrich; Merck KGaA; cat no. 5074)-coated 12-well culture plates, and cultured in PneumaCult ${ }^{\mathrm{TM}}$-Ex Plus Medium (Stemcell Technologies, Inc.; cat. no. \#05040) at $37^{\circ} \mathrm{C}$ in $5 \% \mathrm{CO}_{2}$. To induce inflammation, one part of the adherent epithelial cells were incubated under the following conditions: i) no additions (control); ii) $10 \mu \mathrm{g} / \mathrm{ml}$ LPS for $24 \mathrm{~h}$ (LPS from Pseudomonas aeruginosa; Sigma-Aldrich; Merck KGaA; cat. no. L8643); iii) $10 \mu \mathrm{g} / \mathrm{ml}$ LPS for $24 \mathrm{~h}$, $300 \mu \mathrm{M}$ 2'(3')-O-(4-benzoylbenzoyl)adenosine 5'-triphosphate triethylammonium salt (BzATP) (Sigma-Aldrich; Merck KGaA; cat. no. B6396) was supplemented $1 \mathrm{~h}$ after LPS administration; iv) $20 \mu \mathrm{g} / \mathrm{ml}$ LPS for $24 \mathrm{~h}, 300 \mu \mathrm{M}$ BzATP was supplemented $1 \mathrm{~h}$ after LPS administration. At the end of the incubation, the cells were collected, centrifuged and frozen at $-80^{\circ} \mathrm{C}$ until use. To confirm the role of the $\mathrm{P} 2 \mathrm{X} 7$ receptor in the inflammatory response of HNECs, the other part of the adherent epithelial cells were treated with LPS and A740003 (P2X7 receptor blocker) were incubated under the following conditions: i) no additions (control); ii) $10 \mu \mathrm{g} / \mathrm{ml} \mathrm{LPS} \mathrm{for} 24 \mathrm{~h}$, $300 \mu \mathrm{M}$ BzATP was added $1 \mathrm{~h}$ after LPS administration; iii) $10 \mu \mathrm{g} / \mathrm{ml}$ LPS for $24 \mathrm{~h}, 300 \mu \mathrm{M}$ BzATP was added $1 \mathrm{~h}$ after LPS administration, with supplementation with A740003 (10 $\mu \mathrm{M}$; MedChemExpress; cat no. HY-50697) $15 \mathrm{~min}$ before BzATP stimulation. At the end of the incubation, the supernatants and cells were collected, centrifuged and frozen at $-80^{\circ} \mathrm{C}$ until use.

$W B$. NP specimens were collected and stored in liquid nitrogen at $-80^{\circ} \mathrm{C}$ until use. Total cellular protein was extracted from NP tissue and cultured cells using RIPA lysis buffer (Beyotime Institute of Biotechnology) according to the manufacturer's instructions, and the protein concentration was measured using a BCA protein assay kit (Beyotime Institute of Biotechnology). Then, 12\% SDS-polyacrylamide gels were used to separate the protein $(30 \mu \mathrm{g})$, after which the proteins were transferred onto polyvinylidenedifluoride membranes (Bio-Rad Laboratories, Inc.). The membranes were blocked with $5 \%$ non-fat milk at $4^{\circ} \mathrm{C}$ for $1 \mathrm{~h}$ and then incubated with the working dilution of primary antibodies: Rabbit anti-human P2X7R (1:1,000; GeneTex; cat no. GTX16827); rabbit anti-human NLRP3 (1:1,000; Abcam; cat no. ab260017); mouse anti-human IL-1 $\beta$ (1:5,000; Arigo Biolaboratories; cat. no. ARG66285); rabbit anti-human GAPDH (1:5,000; AntGene; cat. no. ANT012) 
Table I. Characteristics of included subjects.

\begin{tabular}{|c|c|c|c|c|}
\hline Characteristics & ECRSwNP & nECRSwNP & Controls & P-value ${ }^{a}$ \\
\hline Subjects, $\mathrm{n}$ & 16 & 16 & 16 & N/A \\
\hline Sex, male, n (\%) & $11(68.75)$ & $14(87.5)$ & $12(75)$ & 0.394 \\
\hline Age, years, median (IQR) & $50(24.26-66.25)$ & $37.5(26.25-63)$ & $36.5(27.5-48.75)$ & 0.926 \\
\hline With smoking exposure, $\mathrm{n}(\%)$ & $8(50)$ & $10(62.5)$ & $10(62.5)$ & 0.722 \\
\hline With allergic rhinitis, n (\%) & $6(37.5)$ & $4(25)$ & $1(6.25)$ & 0.704 \\
\hline With asthma, n (\%) & $2(12.5)$ & 0 & 0 & 0.484 \\
\hline Prior sinus surgery & $3(18.75)$ & $1(6.25)$ & 0 & 0.6 \\
\hline VAS score, median (IQR) & $11.5(9.25-16)$ & $9.5(7.25-11.75)$ & $3(3-4.75)$ & 0.403 \\
\hline CT score, median (IQR) & $17.5(12.5-20)$ & $15.5(12.5-18)$ & $7(6-8)$ & 0.402 \\
\hline
\end{tabular}

${ }^{a}$ ECRSwNP vs. nECRSwNP. ECRSwNP, eosinophilic chronic rhinosinusitis with nasal polyps; nECRSwNP, non-eosinophilic chronic rhinosinusitis with nasal polyps; VAS, visual analog scale; N/A, not applicable; CT, computed tomography; IQR, interquartile range. Active or passive smoking history $>1$ year.

at $4^{\circ} \mathrm{C}$ overnight. Subsequently, the membranes were washed in Tris-buffered saline mixed with $0.1 \%$ Tween four times for $10 \mathrm{~min}$ each, and then incubated with the appropriate horseradish peroxidase-conjugated secondary antibody: HRP goat anti-rabbit IgG (H+L) (1:4,000; cat. no. ANT020; AntGene) and HRP goat anti-mouse IgG (H+L) (1:4,000; cat. no. ANT019; AntGene) for $1 \mathrm{~h}$ at room temperature. The immunolabelled proteins were detected using BeyoECL Plus (Beyotime Institute of Biotechnology). The gel images were analysed using Quantity One software (version 4.6; Bio-Rad Laboratories, Inc.) to estimate the relative quantitative density of the protein bands. GAPDH was used as an internal control.

Real-time PCR. The total mRNA of primary HNECs was extracted using an E.Z.N.A ${ }^{\mathrm{TM}}$ Total RNA kit (Omega Bio-Tek, Inc.) according to the manufacturers' instructions. A PrimeScript $^{\mathrm{TM}}$ RT Reagent kit with a gDNA Eraser (Takara Biotechnology Co., Ltd.) was used to conduct the reverse transcription reaction $\left(37^{\circ} \mathrm{C}\right.$ for $15 \mathrm{~min}, 85^{\circ} \mathrm{C}$ for $5 \mathrm{sec}$ and stop at $4^{\circ} \mathrm{C}$ ) to obtain cDNA for real-time PCR analysis. Real-time PCR was performed using SYBR ${ }^{\circledR}$ Premix Ex Taq $^{\text {TM }}$ II (TliRNaseH Plus) (X2 concentration) X1 (Takara Biotechnology Co., Ltd.) according to the manufacturer's instructions. Sequence-specific primers for P2X7R, IL-1 $\beta$, and GADPH were as follows: P2X7R, 5'-TCTGTACTTTGC AGCCAATCAGAAC-3' (forward primer); P2X7R, 5'-CCA ACTCTAGTGACCAAACCAGGAA-3' (reverse primer); IL-1 $\beta$, 5'-CCAGGGACAGGATATGGAGCA-3' (forward primer); IL-1 $\beta$, 5'-TTCAACACGCAGGACAGGTACAG-3' (reverse primer); GAPDH, 5'-GCACCGTCAAGGCTGAGA AC-3' (forward primer), GAPDH, 5'-TGGTGAAGACGC CAGTGGA-3' (reverse primer). The real-time PCR protocol was as follows: Denaturation at $95^{\circ} \mathrm{C}$ for $30 \mathrm{sec}$, followed by 40 cycles of amplification at $95^{\circ} \mathrm{C}$ for $5 \mathrm{sec}, 60^{\circ} \mathrm{C}$ for $30 \mathrm{sec}$ and annealing at $60^{\circ} \mathrm{C}$ for $30 \mathrm{sec}$. Relative mRNA level was determined by the $2^{-\Delta \Delta \mathrm{Cq}}$ method (20).

Enzyme-linked immunosorbent assay (ELISA). Human IL-1 $\beta$ was measured in cell culture supernatants from HNECs under different culture conditions using an ELISA kit (Arigo; cat. no. ARG80101) following the manufacturers' instructions. Samples were run at least in duplicate.

Statistical analysis. For continuous clinical variables, the data are expressed as median and interquartile ranges, or as box and whisker plots displaying medians and interquartile ranges, which were analysed by the Kruskal-Wallis H-test and Mann-Whitney U test. For dichotomous parameters, the $\chi^{2}$ test or Fisher's exact test was performed to determine the difference between groups. Data were analysed using the Statistical Package for the Social Sciences (version 22.0; SPSS Inc.). For tissue samples and in vitro experiments, the data are expressed as mean \pm standard deviation and were analysed by one-way ANOVA. Tukey's post hoc test was also performed. $\mathrm{P}<0.05$ was considered to indicate a statistically significant difference.

\section{Results}

Histological changes and localisation of $P 2 X 7 R$ in nasal mucosa. Overall, all patients, including 16 control subjects and 32 patients with CRSwNP, underwent H\&E staining, which showed that numerous eosinophils infiltrated the nasal mucosa of patients with ECRSwNP. Furthermore, an intense oedematous stroma and subepithelial and perivascular inflammatory cell infiltration was also observed in CRSwNP (Fig. 1A). Immunofluorescence (Fig. 1B) showed that P2X7R was predominantly expressed in epithelial cells. It is worth noting that the level of receptor in the ECRSwNP group was higher compared with that of the other two groups.

Expression of P2X7R is higher in ECRSwNP compared with the control group. Protein content was analysed in the nasal mucosa of ECRSwNP, NECRSwNP and control groups. As shown by WB (Fig. 2A), P2X7R (Fig. 2B), NLRP3 (Fig. 2C) and IL-1 $\beta$ (Fig. 2D) were significantly overexpressed in CRSwNP $(\mathrm{P}<0.05)$, especially in the ECRSwNP group. The mRNA expression of P2X7R and IL-1 $\beta$ were elevated in the CRSwNP groups compared with the control group, and these increases were also found in the ECRSwNP group compared with the NECRSwNP group (Fig. 2E and F). 
A

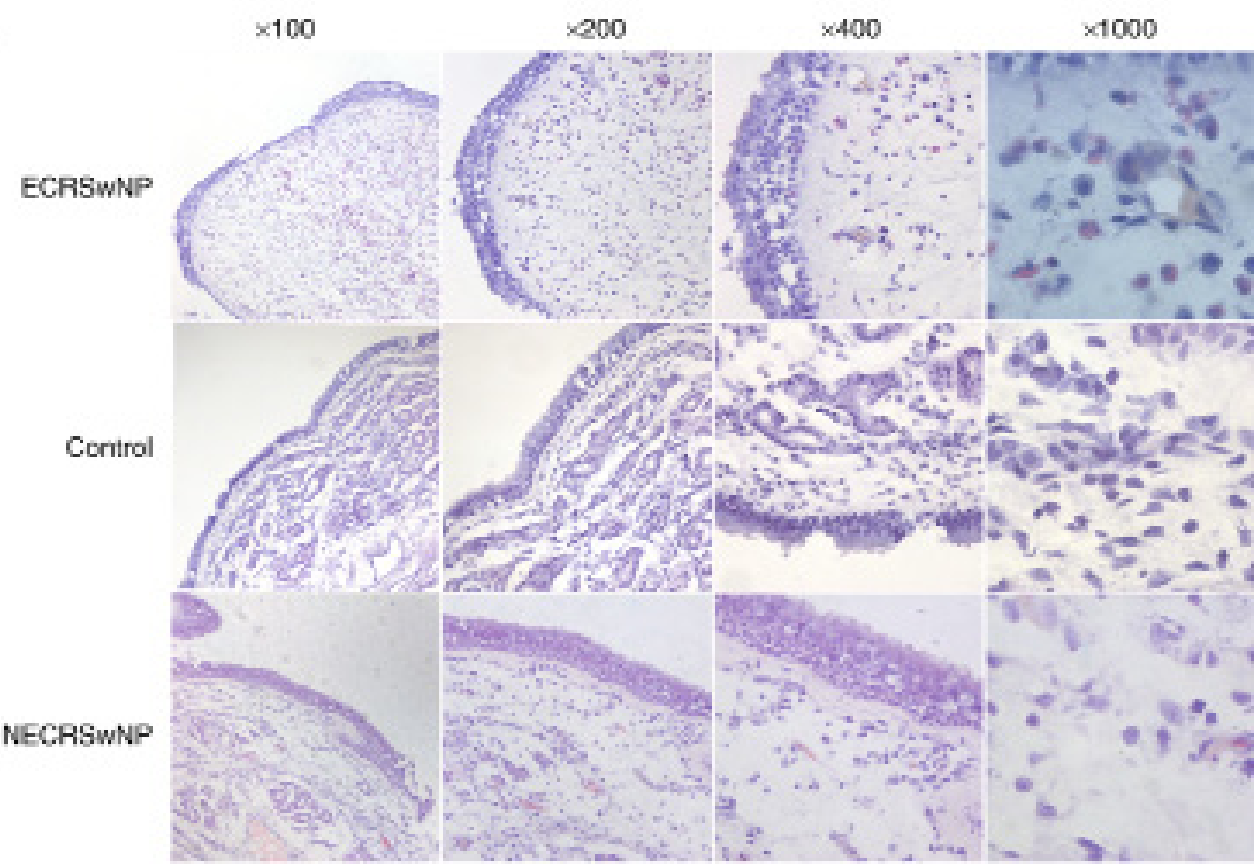

\section{B}
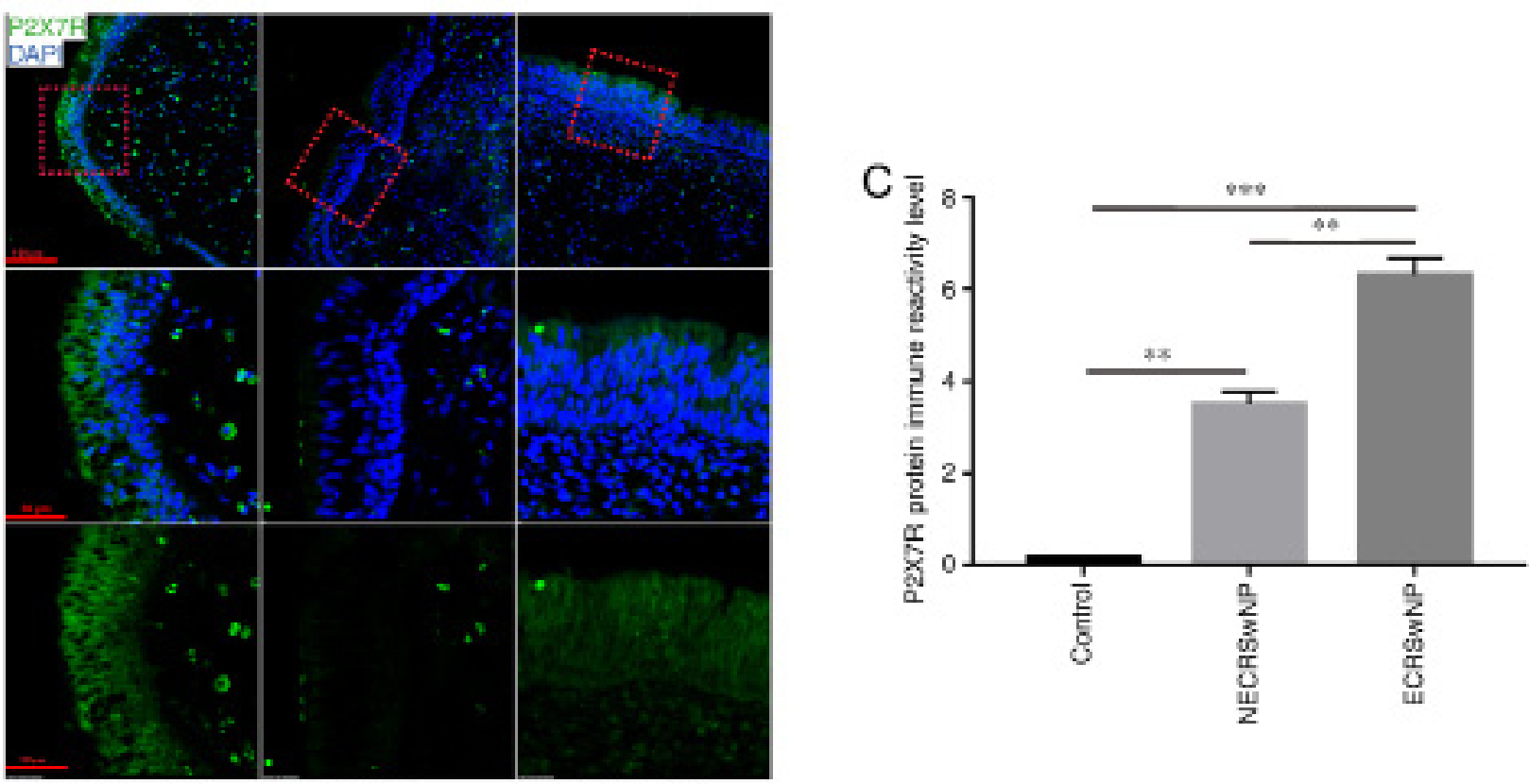

ECASWNP

Contral

NECASWNP

Figure 1. Haematoxylin and eosin staining of nasal mucosa and immunolabelling of P2X7R. Numerous eosinophils infiltrated the nasal mucosa of ECRSwNP. (A) Immunofluorescence staining showed that P2X7R was predominantly expressed in epithelial cells and P2X7R protein levels were significantly overexpressed in CRSwNP groups, especially in the ECRSwNP group vs. control group. (B and C) Data represent means \pm SD. ${ }^{* * *} \mathrm{P}<0.01$ and ${ }^{* * *} \mathrm{P}<0.001$. CRSwNP, chronic rhinosinusitis with nasal polyps; ECRSwNP, eosinophilic CRSwNP; P2X7R, purinergic 2X7 receptor.

IL-1 $\beta$ is upregulated after incubation with LPS combined with BzATP. LPS induces a cellular inflammatory response in vitro. The expression of IL-1 $\beta$ was evaluated under different conditions by WB (Fig. 3). When treated with LPS alone, the expression of IL-1 $\beta$ increased relative to controls. When LPS was combined with BzATP, IL-1 $\beta$ markedly increased, and the increase was statistically significant compared with the control group $(\mathrm{P}<0.05)$. There was no significant difference in the expression of IL- $1 \beta$ between the $10 \mu \mathrm{g} / \mathrm{ml}$ LPS group and the $20 \mu \mathrm{g} / \mathrm{ml}$ group, thus, $10 \mu \mathrm{g} / \mathrm{ml}$ LPS combined with BzATP was chosen as the inflammatory stimulation condition.

Expression of $P 2 X 7 R$ shows no significant change, but NLRP3 is downregulated after stimulation with A740003. A740003 significantly blocks the sustained phase of the BzATP-induced response. The expression levels of P2X7R and NLRP3 were evaluated by WB (Fig. 4). The expression of P2X7R (Fig. 4B) showed no significant change, while the expression of NLRP3 
A
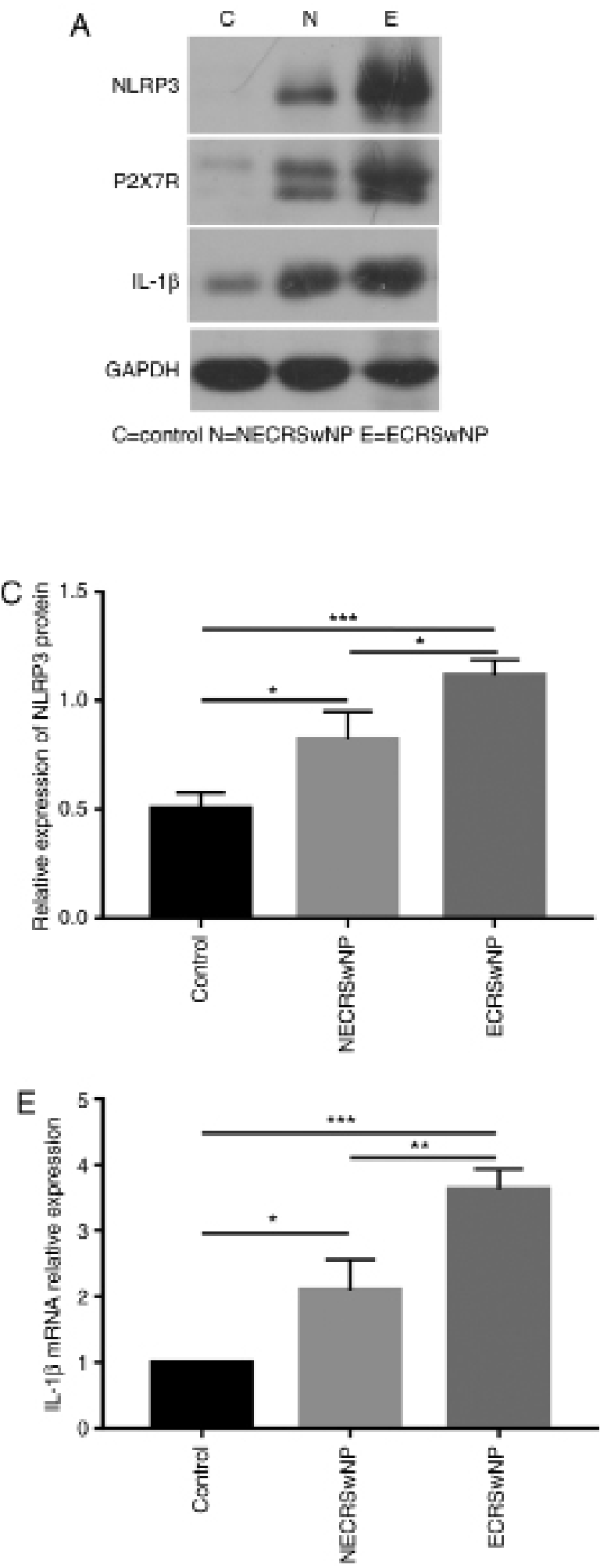

B
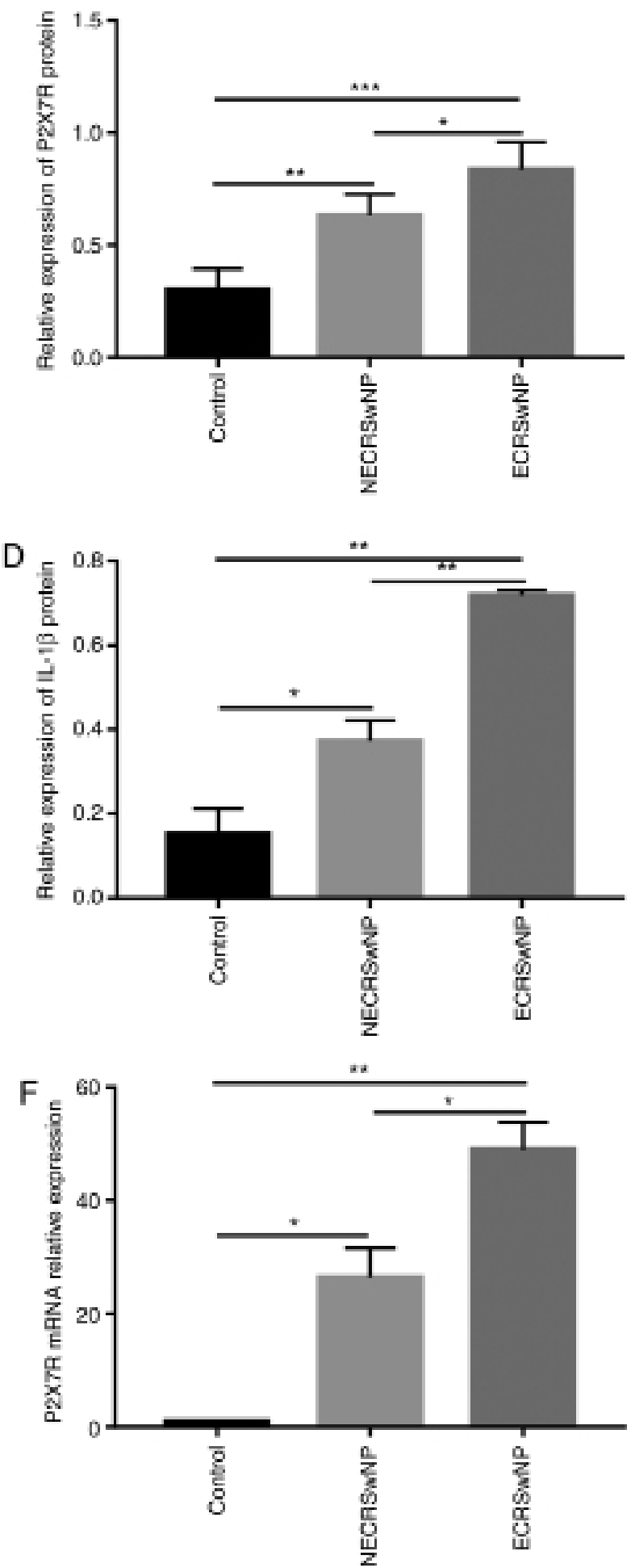

Figure 2. Expression profile of P2X7R in nasal mucosa. (A) Western blotting analysis showed that P2X7R, (B) NLRP3 (C) and IL-1 (D) protein levels were significantly increased in the CRSwNP groups compared with the control group, and were significantly more highly expressed in the ECRSwNP group compared with the NECRSwNP group. The mRNA expression levels of P2X7R and IL-1 $\beta$ were elevated in the CRSwNP groups compared with the control group, and these increases were also found in the ECRSwNP group compared with the NECRSwNP group. (E and F) ${ }^{*} \mathrm{P}<0.05 ;{ }^{* * *} \mathrm{P}<0.01 ;{ }^{* * * *} \mathrm{P}<0.001$. CRSwNP, chronic rhinosinusitis with nasal polyps; NECRSwNP, non-eosinophilic CRSwNP; GAPDH, glyceraldehyde 3-phosphate dehydrogenase; P2X7R, purinergic $2 X 7$ receptor; NLRP3, NLR family pyrin domain-containing 3; IL-1 $\beta$, interleukin-1 $\beta$.

(Fig. 4C) was significantly downregulated after A740003 treatment in HNECs after stimulation with LPS+BzATP. Similarly, after treatment of HNECs with LPS combined with BzATP, the expression of both P2X7R and IL-1 $\beta$ mRNA increased $(\mathrm{P}<0.05)$. After addition of the inhibitor A74003, compared with the inflammatory stimulation group, the expression of P2X7R decreased somewhat, but this was not statistically significant, while IL-1 $\beta$ mRNA significantly 


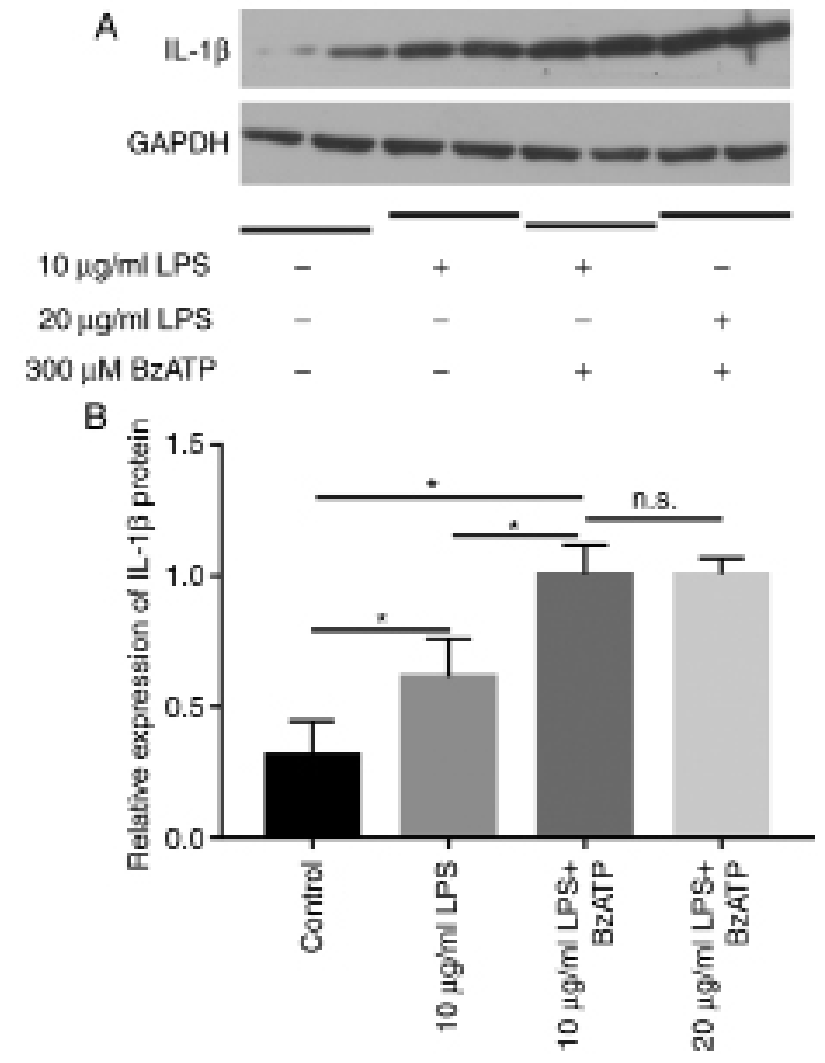

Figure 3. Expression profiles of IL-1 $\beta$ after LPS treatment for $24 \mathrm{~h}$, followed by $300 \mu \mathrm{M}$ BzATP $1 \mathrm{~h}$ after LPS administration. (A and B) IL-1 $\beta$ was found to be significantly increased in cultured human nasal epithelial cells after incubation with LPS+BzATP. Compared with the control group, IL-1 $\beta$ was significantly increased in the $10 \mu \mathrm{g} / \mathrm{ml}$ LPS group and the $20 \mu \mathrm{g} / \mathrm{ml}$ LPS combined with BzATP group, but there was no significant difference between the two groups. ${ }^{*} \mathrm{P}<0.05$. n.s., not statistically significant; LPS, lipopolysaccharides; BzATP, 2'(3')-O-(4-benzoylbenzoyl)adenosine 5'-triphosphate triethylammonium salt; IL-1 $\beta$, interleukin- $1 \beta$.

decreased (Fig. 4D and E). The level of IL-1 $\beta$ in the culture supernatant increased after LPS+BzATP stimulation and was inhibited by A740003 (Fig. 4F), suggesting that the blockade of P2X7R might prevent NLRP3 inflammasome activation.

\section{Discussion}

The present study found that P2X7R, NLRP3 and IL-1 $\beta$ protein levels were significantly increased in the CRSwNP groups compared with the control group and expression was further significantly higher in the ECRSwNP group compared with the NECRSwNP group. Thus, the inflammatory form of ECRSwNP is more severe compared with NECRSwNP. There are obvious regional differences in the inflammatory characteristics of CRS. Approximately $80 \%$ of nasal polyps in Western patients are eosinophilic, while the rate in Asia is $<50 \%$ (21). However, recent studies have shown that the incidence of ECRS in East Asian countries is increasing. A Chinese study showed that the proportion of eosinophilic CRSwNP significantly increased from 59.1 to $73.7 \%$ over 11 years (19). ECRS is considered a special and recalcitrant subtype of CRS (21).

ATP concentration is maintained at a low level in healthy tissues (22). Extracellular ATP is involved in the release of various pro-inflammatory cytokines including thymic stromal lymphopoietin, IL-25 and IL-33 in nasal mucosal inflammation $(23,24)$. In pathological situations, cell injury leads to a substantial increase in extracellular ATP as a key danger alarmin that initiates inflammation and further amplifies immune responses (25). As the effects of extracellular ATP are mediated by $\mathrm{P} 2$ receptors, the role of $\mathrm{P} 2 \mathrm{X} 7$ was investigated, which is the most involved of the $\mathrm{P} 2$ receptors in inflammation and infection and exhibits a high binding affinity for ATP (22).

In the present study, P2X7 was expressed in epithelial cells in control subjects; this result was consistent with previous reports (16). Furthermore, it was found that the expression of P2X7R was increased in ECRSwNP compared with controls and the protein was mainly located in epithelial cells. Since P2X7R has been demonstrated to be highly expressed in immune cells, the increase in P2X7R expression may be partly associated with enhanced infiltration of macrophages into nasal polyps (26). It was also found that LPS increased the expression level of IL-1 $\beta$ in a dose-dependent manner when combined with BzATP in primary human HNECs. LPS stimulation can result in the accumulation of cytoplasmic IL-1 $\beta$ through Toll-like receptor 4 (27). Meanwhile, LPS may cause pannexin-1 opening, allowing ATP release, which then triggers $\mathrm{K}^{+}$efflux, to promote inflammasome-mediated caspase-1 activation. The activated NLRP3 inflammasome catalyses pro-IL-1 $\beta$ cleavage. NLRP3 inflammasomes have been proven to be activated in both ECRSwNP and NECRSwNP (9) and the present study findings confirmed this. The downstream inflammatory cytokines released after NLRP3 inflammasome assembly include IL-1 $\beta$ and IL-18, which were elevated in CRSwNP (9). Elevated IL-1 $\beta$ expression was found in HNECs after LPS inoculation. Further examination using the P2X7-selective antagonist A740003 confirmed that the LPS-induced effects are P2X7 specific. A740003 is a selective competitive antagonist of P2X7R (28). In addition, A740003 can block P2X7R-mediated calcium influx, pore formation and IL-1 $\beta$ release (29). This effect was also found in HNECs in the present study. This suggested that P2X7R may be implicated in the release of IL-1 $\beta$ in CRSwNP via the activation of the NLRP3 inflammasome (Fig. 5). P2X7R expression after antagonist administration in HNECs was slightly decreased compared with LPS alone. This effect may be associated with membrane internalization stimulated by P2X7R under inflammatory conditions. The administration of antagonists could decrease P2X7R activation and membrane internalization, which could induce receptor relocation, degradation and replenishment in the membrane, resulting in decreased receptor expression (30).

Nasal epithelial cells exist at the surface of the nasal mucosa and are capable of detecting microbial products and endogenous molecules associated with cellular damage via an assortment of pattern recognition receptors (31). NLRP3, a member of the NLR family, induces an increase in the expression of IL-1 $\beta$, and can be activated by pathogens (9). The present study found that after blocking P2X7R, the expression of NLRP3 decreased, indicating that P2X7R is upstream of NLRP3 in nasal polyps. When persistent inflammation in nasal mucosa results in tissue damage, a variety of molecules are released that are normally sequestered intracellularly or 
A

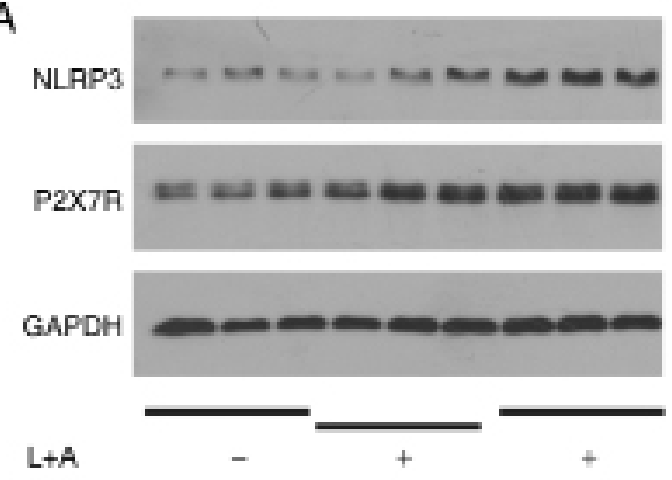

74

L-LPS, A-BzATP, 74-A740003

C

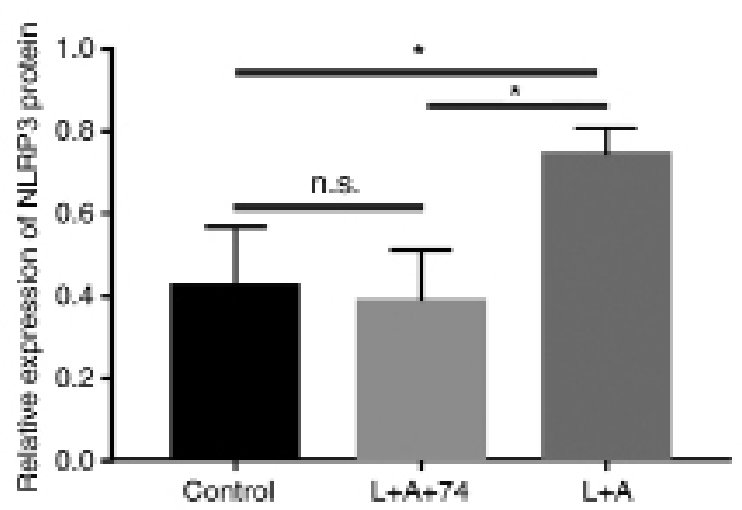

E

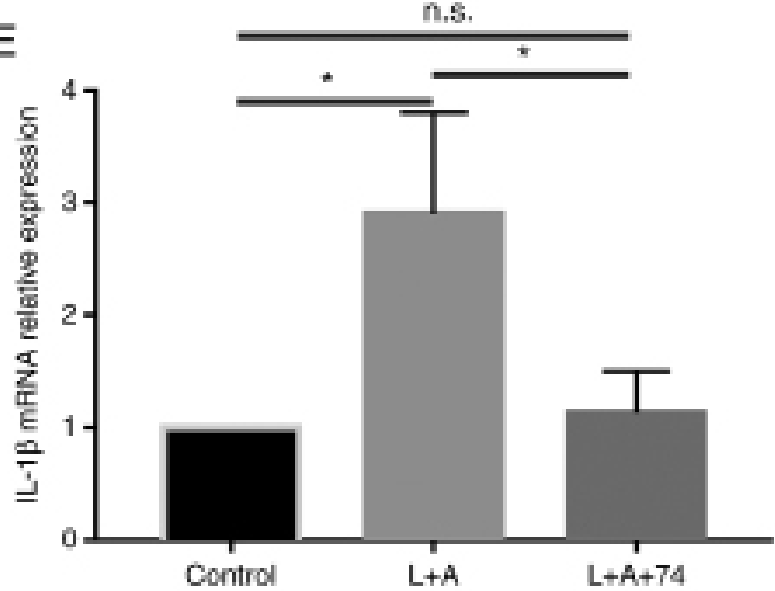

B

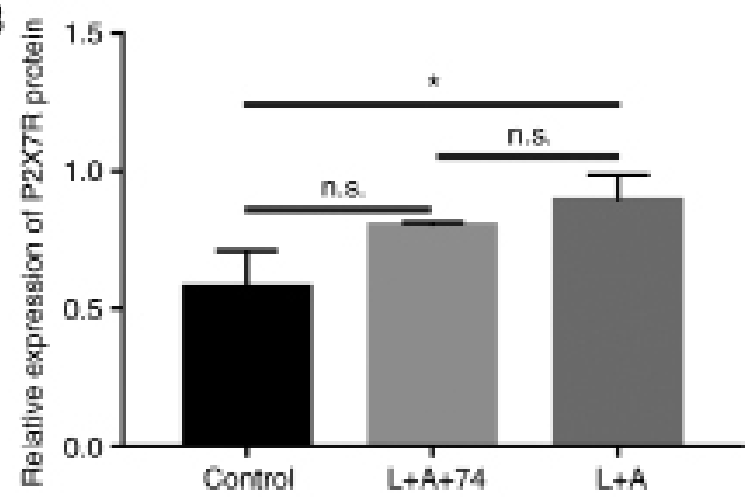

D

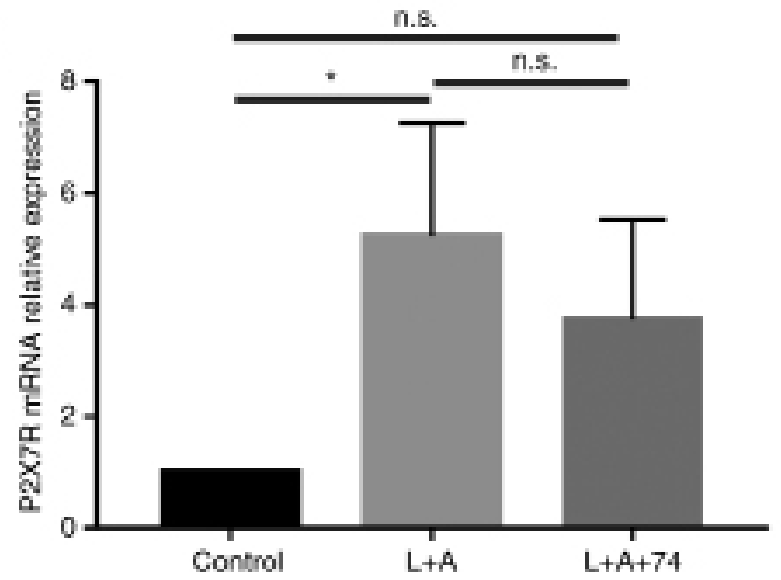

F

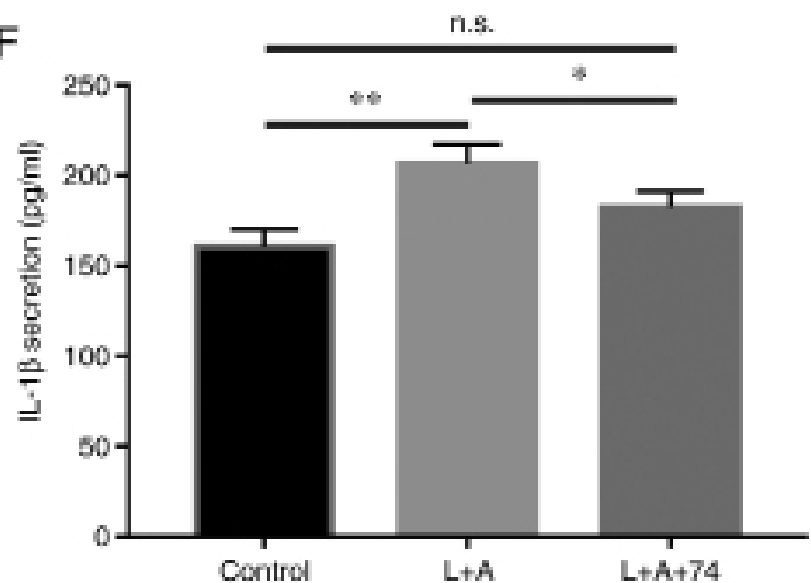

Figure 4. Inflammatory stimulation with LPS combined with BzATP was inhibited by A740003. (A) Western blot analysis of the expression levels of P2X7R (B) and NLRP3 (C) in HNEC after A740003 inhibition of inflammation induced by LPS+BzATP. The expression of P2X7R and NLRP3 was significantly increased in response to LPS plus BzATP; furthermore, the selective P2X7R antagonist, A740003, suppressed this process, decreasing the protein expression of NLRP3 and P2X7R. (D and E) The mRNA expression levels of (D) P2X7R and (E) IL-1 $\beta$ were decreased after stimulation with LPS+BzATP and subsequent inhibition with A740003. (F) Enzyme-linked immunosorbent assay results show the changes in the concentration of IL-1 $\beta$ in the cell culture supernatant. ${ }^{*} \mathrm{P}<0.05$ and ${ }^{* *} \mathrm{P}<0.01$. NLRP3, NLR family pyrin domain-containing 3; LPS, lipopolysaccharides; BzATP, 2'(3')-O-(4-benzoylbenzoyl)adenosine 5'-triphosphate triethylammonium salt; IL-1 $\beta$, interleukin-1 $\beta$; P2X7R, purinergic 2 X7 receptor.

within the extracellular matrix. The nasal mucosa is exposed to harmful elements, such as pathogens and air pollutants, leading to an enhanced and sustained stream of danger signals $(32,33)$. Studies examining the nasal mucosa of patients with CRSwNP have demonstrated the infiltration of immune cells including $\mathrm{CD}^{+} \mathrm{T}$-cells, eosinophils, neutrophils and macrophages, and such inflammatory cells have been implicated in the pathogenesis of CRSwNP (3). Therefore, it was speculate that P2X7R may regulate the occurrence and development of nasal polyps by activating the NLRP3 inflammasome and mediating the release of IL-1 $\beta$.

There are some limitations to the present study, which should be pointed out. Firstly, the experiment only included 32 patients with CRSwNP and 16 control subjects, thus the 


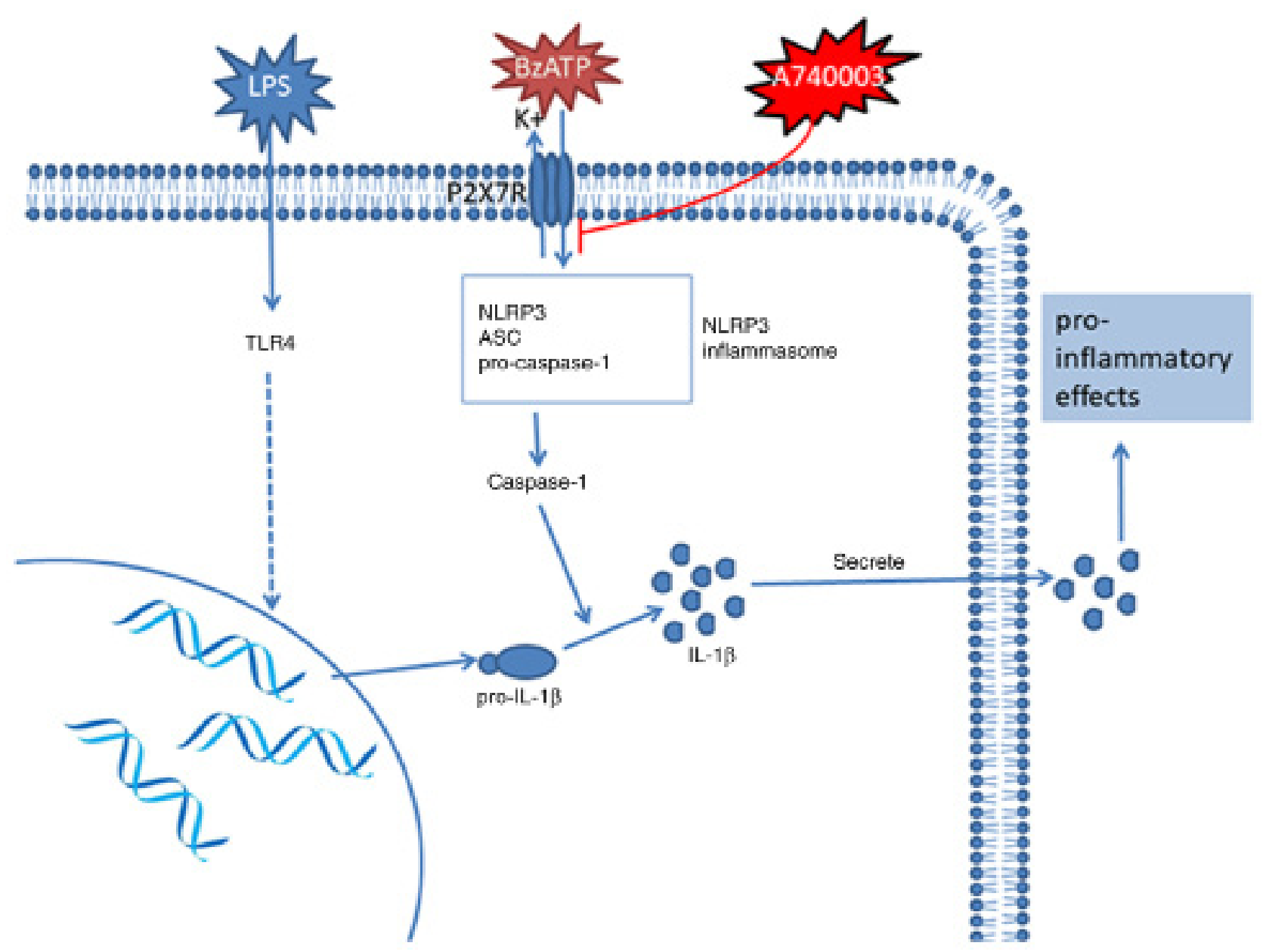

Figure 5. LPS activates Toll-like receptor 4, leading to transcription of the IL-1 $\beta$ gene and translation of the 31-kDa pre-cytokine (pro-IL-1 $\beta$ ). P2X7R exposure to its agonist, BzATP, triggers depolarization via the opening of cation channels, which triggers $\mathrm{K}^{+}$efflux, leading to stimulation of the NLRP3 inflammasome. Activated caspase-1 converts pro-IL-1 $\beta$ to the $17-\mathrm{kDa}$ mature protein (IL-1 $\beta$ ), which is then secreted to the extracellular space. A740003, a selective competitive antagonist of P2X7R, blocks P2X7R-mediated calcium influx, pore formation and IL-1 $\beta$ release. TLR4, Toll-like receptor 4; IL-1 $\beta$, interleukin-1 $\beta$; P2X7R, purinergic 2 X7 receptor; BzATP, 2'(3')-O-(4-benzoylbenzoyl)adenosine 5'-triphosphate triethylammonium salt; NLRP3, NLR family pyrin domain-containing 3; ASC, apoptosis-associated speck-like protein containing a CARD.

sample size is small. A larger sample is needed to confirm the present findings. Secondly, the experiment only compared patients with CRSwNP with a control group; consequently the role of P2X7R in the pathogenesis of CRSsNP and chronic refractory rhinosinusitis needs further research and analysis.

In summary, the present study found that the expression of P2X7R in the nasal mucosa of patients with CRSwNP and HNECs under inflammatory conditions was higher compared with that of the control group. P2X7R-NLRP3 inflammasome signaling pathway can be augmented by LPS combined with BzATP, but suppressed by A740003.

\section{Acknowledgements}

Not applicable.

\section{Funding}

This work was supported by the National Natural Science Foundation of China (grant nos. 81570898 and 18020067) and Health Commission of Hubei Province Scientific research project (no. WJ2021M250).

\section{Availability of data and materials}

The datasets used and/or analysed during the current study are available from the corresponding author on reasonable request.

\section{Authors' contributions}

YW and SC performed the majority of the experiments, the statistical analysis and prepared the manuscript. WW and JC performed experiments and helped to draft the manuscript. WW, JC and YJW analysed the data. WK and YJW participated in the conception and design of the study. YW and YJW confirm the authenticity of all the raw data. All authors read and approved the final manuscript.

\section{Ethics approval and consent to participate}

The present study was approved by the ethical committees of Tong-ji Medical College, Huazhong University of Science and Technology (permit no. S135). All participants of the study were informed and signed a consent form. 


\section{Patient consent for publication}

Not applicable.

\section{Competing interests}

The authors declare that they have no competing interests.

\section{References}

1. Fokkens WJ, Lund VJ, Hopkins C, Hellings PW, Kern R, Reitsma S, Toppila-Salmi S, Bernal-Sprekelsen M, Mullol J, Alobid I, et al: European position paper on rhinosinusitis and nasal polyps 2020. Rhinology 58 (Suppl S29): S1-S464, 2020.

2. Yim MT and Orlandi RR: Evolving Rhinology: Understanding the burden of chronic rhinosinusitis today, tomorrow, and beyond. Curr Allergy Asthma Rep 20: 7, 2020.

3. Zhang Y, Gevaert E, Lou H, Wang X, Zhang L, Bachert C and Zhang N: Chronic rhinosinusitis in Asia. J Allergy Clin Immunol 140: 1230-1239, 2017.

4. Lourijsen ES, Fokkens WJ and Reitsma S: Direct and indirect costs of Dutch adult patients with Chronic Rhinosinusitis with nasal polyps. Rhinology 58: 213-217, 2020.

5. Czerny MS, Namin A, Gratton MA and Antisdel JL: Histopathological and clinical analysis of chronic rhinosinusitis by subtype. Int Forum Allergy Rhinol 4: 463-469, 2014.

6. Van Zele T, Holtappels G, Gevaert P and Bachert C: Differences in initial immunoprofiles between recurrent and nonrecurrent chronic rhinosinusitis with nasal polyps. Am J Rhinol Allergy 28: 192-198, 2014.

7. Calus L, Van Bruaene N, Bosteels C, Dejonckheere S, Van Zele T, Holtappels G, Bachert C and Gevaert P: Twelve-year follow-up study after endscopic sinus surgery in patients with chronic rhinosinusitis with nasal polyposis. Clin Transl Allergy 9: 30, 2019.

8. DeConde AS, Mace JC, Levy JM, Rudmik L, Alt JA and Smith TL: Prevalence of polyp recurrence after endoscopic sinus surgery for chronic rhinosinusitis with nasal polyposis Laryngoscope 127: 550-555, 2017.

9. Lin H, Li ZP, Lin D, Zheng CQ and Zhang WT: Role of NLRP3 inflammasome in eosinophilic and non-eosinophilic chronic rhinosinusitis with nasal polyps. Inflammation 39: 2045-205, 2016.

10. Wang YJ, Gong GQ, Chen S, Xiong LY, Zhou XX, Huang X and Kong WJ: NLRP3 inflammasome sequential changes in Staphylococcus aureus-induced mouse model of acute rhinosinusitis. Int J Mol Sci 15: 15806-15820, 2014.

11. Yazdi AS, Guarda G, Riteau N, Drexler SK, Tardivel A, Couillin I and Tschopp J: Nanoparticles activate the NLR pyrin domain containing 3 (Nlrp3) inflammasome and cause pulmonary inflammation through release of IL- $1 \alpha$ and IL-1 $\beta$. Proc Natl Acad Sci USA 107: 19449-19454, 2010.

12. Okano M, Fujiwara T, Makihara S, Fujiwara R, Higaki T, Kariya S, Noda Y, Haruna T and Nishizaki K: Characterization of IL-18 expression and release in the pathogenesis of chronic rhinosinusitis. Int Arch Allergy Immunol 160: 275-286, 2013.

13. Robbins GR, Wen H and Ting JP: Inflammasomes and metabolic disorders: Old genes in modern diseases. Mol Cell 54: 297-308, 2014.

14. Dostert C, Pétrilli V, Bruggen RV, Steele C, Mossman BT and Tschopp J: Innate immune activation through Nalp3 inflammasome sensing of asbestos and silica. Science 320: 674-677, 2008

15. Qu Y, Franchi L, Nunez G and Dubyak GR: Nonclassical IL-1 beta secretion stimulated by $\mathrm{P} 2 \mathrm{X} 7$ receptors is dependent on inflammasome activation and correlated with exosome release in murine macrophages. J Immunol 179: 1913-1925, 2007.

16. Kim CH, Kim SS, Choi JY, Shin JH, Kim JY, Namkung W, Lee JG, Lee MG and Yoon JH: Membrane-specific expression of functional purinergic receptors in normal human nasal epithelial cells. Am J Physiol Lung Cell Mol Physiol 287: L835-L842, 2004.
17. Di Virgilio F: Liaisons dangereuses: $\mathrm{P} 2 \mathrm{X}(7)$ and the inflammasome. Trends Pharmacol Sci 28: 465-472, 2007.

18. Liao B, Cao PP, Zeng M, Zhen Z, Wang H, Zhang YN, Hu CY, Ma J, Li ZY, Song J, et al: Interaction of thymic stromal lymphopoietin, IL-33, and their receptors in epithelial cells in eosinophilic chronic rhinosinusitis with nasal polyps. Allergy 70: 1169-1180, 2015.

19. Wang WQ, Gao YL, Zhu ZZ, Zha Y, Wang XW, Qi F, Zhou LG, Pang JY, Gao ZQ and Lv W: Changes in the clinical and histological characteristics of Chinese chronic rhinosinusitis with nasal polyps over 11 years. Int Forum Allergy Rhinol 9: 149-157, 2019.

20. Livak KJ and Schmittgen TD: Analysis of relative gene expression data using real-time quantitative PCR and the 2(-Delta Delta C(T)) method. Methods 25: 402-408, 2001.

21. Wang ET, Zheng Y, Liu PF and Guo LJ: Eosinophilic chronic rhinosinusitis in East Asians. World J Clin Cases 2: 873-882, 2014.

22. Lohman AW, Billaud M and Isakson BE: Mechanisms of ATP release and signalling in the blood vessel wall. Cardiovasc Res 95: 269-280, 2012.

23. Kato T, Kouzaki H, Matsumoto K, Hosoi J and Shimizu T: The effect of calprotectin on TSLP and IL-25 production from airway epithelial cells. Allergol Int 66: 281-289, 2017.

24. Paris G, Pozharskaya T, Asempa T and Lane AP: Damage-associated molecular patterns stimulate interleukin-33 expression in nasal polyp epithelial cells. Int Forum Allergy Rhinol 4: 15-21, 2014.

25. Cauwels A, Rogge E, Vandendriessche B, Shiva S and Brouckaert P: Extracellular ATP drives systemic inflammation, tissue damage and mortality. Cell Death Dis 5: e1102, 2014.

26. Wang ZC, Yao Y, Wang N, Liu JX, Ma J, Chen CL, Deng YK, Wang MC, Liu Y, Zhang XH and Liu Z: Deficiency in interleukin-10 production by M2 macrophages in eosinophilic chronic rhinosinusitis with nasal polyps. Int Forum Allergy Rhinol 8: 1323-1333, 2018.

27. Weigt SS, Palchevskiy V and Belperio JA: Inflammasomes and IL-1 biology in the pathogenesis of allograft dysfunction. J Clin Inves 127: 2022-2029, 2017

28. Nelson DW, Gregg RJ, Kort ME, Perez-Medrano A, Voight EA, Wang Y, Grayson G, Namovic MT, Donnelly-Roberts DL, Niforatos W, et al: Structure-activity relationship studies on a series of novel, substituted 1-benzyl-5-phenyltetrazole P2X7 antagonists. J Med Chem 49: 3659-3666, 2006.

29. Donnelly-Roberts DL and Jarvis MF: Discovery of P2X7 receptor-selective antagonists offers new insights into $\mathrm{P} 2 \mathrm{X} 7$ receptor function and indicates a role in chronic pain states. $\mathrm{Br}$ J Pharmacol 151: 571-579, 2007.

30. Feng YH, Wang LQ, Wang QF, Li X, Zeng RB and Gorodeski GI: ATP stimulates GRK-3 phosphorylation and beta-arrestin-2-dependent internalization of $\mathrm{P} 2 \mathrm{X} 7$ receptor. Am J Physiol Cell Physiol 288: C1342-C1356, 2005.

31. Stevens WW, Schleimer RP and Kern RC: Chronic rhinosinusitis with nasal polyps. J Allergy Clin Immunol Pract 4: 565-572, 2016.

32. Gallucci S and Matzinger P: Danger signals: SOS to the immune system. Curr Opin Immunol 13: 114-119, 2001.

33. Heffler E, Malvezzi L, Boita M, Brussino L, De Virgilio A, Ferrando M, Puggioni F, Racca F, Stomeo N, Spriano G and Canonica GW: Immunological mechanisms underlying chronic rhinosinusitis with nasal polyps. Expert Rev Clin Immunol 14: 731-737, 2018.

This work is licensed under a Creative Commons Attribution-NonCommercial-NoDerivatives 4.0 International (CC BY-NC-ND 4.0) License. 